\title{
Biochips for Physical Exercise Studies
}

\author{
Neşe Akpınar Kocakulak *1 (iD), İbrahim Ünal ${ }^{2}$ (iD)
}

\begin{abstract}
In early 1990s, microfluidics technology was mainly aiming at the manipulation of fluids in micro-scale and nanoscale. At present, with the development of microfluidics, it has been widely used in the life science and medical researches with significant achievements. The microfluidics technology can be used in single cell capture, cell screening, and synthesis of biomacromolecules. Some microfluidic chips have already been commercialized and applied in disease detection, drug delivery and bioscience. However, the physical index oriented wearable technology ignored another part of the most important indications in health monitoring i.e. the body fluid. The body fluid in this review refers to the blood, sweat, interstitial fluid, saliva, tears, and urine. The current medical procedures for the testing of body fluid involve using highly sophisticated instrument such as atomic absorption spectrometry, ion chromatography and gas chromatograph for the detection of specific targets in body fluid. For correct detection of changes in body fluids, it is necessary to intervene in body fluids naturally. Physical fatigue is known to have a direct effect on body fluids. For this reason, microfluidic chips are used in experiments after exercise. Also exercise; diabetes, cancer, cardiovascular disease, muscle, immune, and age-related decline in cognitive function have been documented against the protect. In addition, regular physical exercise is the most powerful initiative known to have positive effects on health and aging.

In this review, the production and recent developments of microfluidic chips was classified by the detection object of the body fluid.

\section{Keywords}

Microfluidics devices; microfabrication; microfluidics; microchips; biochips.

${ }^{1}$ Izmir Democracy University, Faculty of Health Sciences, Department of Sports Sciences

2 Izmir Democracy University, Institute of Sciences, Department of Biomedical Sciences and Engineering

*Corresponding Author: nese.kocakulak@idu.edu.tr

Manuscript received date: June 14, 2019

Accept Date: June 28, 2019

Published Date: June 30, 2019.
\end{abstract}

\section{INTRODUCTION}

Miniaturization is one of the key driving factors in the evolution of modern technology [1,2]. It enables devices to have complex functionalities in a smaller volume, with less energy consumption and lower costs per implemented function. Other correlated benefits from miniaturization include faster processing time, lower manufacturing and operational costs, smaller volumes of needed samples and reactants, integration with other devices (multifunctionality), increased safety and reliability by having less external interconnects between the different parts, and high throughput [3]. Miniaturization brings molecules or samples closer together for more effective, efficient, and rapid interactions [4]. Miniaturization also increases the portability of microfluidic devices because the devices are compact yet fully functional by themselves [4].

Microfluidics allows for handling of fluid with volumes typically in the range of nano- to microliters $\left(10^{-9}\right.$ to $\left.10^{-6} \mathrm{~L}\right)$ or smaller [3,4]. The attractive and advantageous characteristics of microfluidics include down-scaling and miniaturization. Microfluidics utilizes and consumes less fluid volumes, and requires fewer materials to make the actual device, making the device and procedures more cost-effective and capable of being mass produced [5, 6]. The downsizing of microfluidic channels results in faster analysis and response times, owing to higher surface to volume ratios, shorter diffusion distances, and smaller heating capacities [4].

Several new microsystems have been created by the fabrication and integration of small electrical and mechanical components put together as a system, using techniques that are inherited from or related to the integrated circuit technologies (IC) [7]. On of these, microelectromechanical systems (MEMS) are precise, miniaturized systems with primary functionalities in 
both electrical and mechanical domains, with applications covering a large range of phenomena in the physical, chemical, and biological domains [3]. MEMS devices open the possibility of cointegration in a single complex system of functions like sensing, electronic signal processing, and electromechanical actuation that are traditionally performed by distinctly separated and bulky functional blocks. Depending on the specific domain of functionality and application of these devices, different terms have been coined to categorize them. As examples, RF MEMS devices are used for radio frequency telecommunication applications (e.g., switches, transmission lines, and antennas) [8], optical MEMS refers to devices interacting with light (e.g., image scanner micro mirrors, and optical switches) [9, 10] and bio-MEMS [11]/microfluidics [12] are used in systems applied to biological assays and fluid manipulation (e.g., body fluids and cell manipulation, DNA analysis [13], toxins detection, diseases diagnosis and treatment, and drug delivery [14]).

The experience accumulated for the microfluidic chips in the life science and medical research recently opened a new gate for the wearable technology, i.e. wearable microfluidics $[15,16]$. As an emerging technology of life science, electronics, material science and chemistry, wearable technology has experienced a significant development over the past few years. Wearable technology has been extensively used in life monitoring [17, 18], disease detection [19, 20], sports science [21, 22] and military [23], etc. Wearable technology refers to the certain kind of equipment that can be directly wore or integrated in the cloths and accessories.Traditional wearable technology aimed at detection of physical index such as heart rate [24], body temperature [25], motion tracking [26], bioelectricity signal [27] and steps [28] using photoelectric detection method [29].

\subsection{Microfluidics}

Microfluidics is the field of the microsystems technology that deals with the study and control of fluids in hydraulically/geometrically small systems, with lengths typically in the range of a few micrometers up to a few millimeters or centimeters $[3,4,30]$. It is an enabling technology to perform biological and chemical experiments at greatly reduced spatial scales, with minimal material consumption and high-throughput. It is based on the control of flows in microchannels with characteristic dimensions ranging from millimeters to micrometers, constituting the basis of technologies known as micro total analysis systems ( $\mu$ TAS) or labs-on-a-chip (LOC) [4].

Microfluidics is key to advancing molecular sensors based on bioassays including immunoassay, cell separation, DNA amplification, and analysis, among many other examples. Microfluidic systems process a large number of parallel experiments rapidly with a small amount of reagent and automate chemical, biological, and medical applications on a large scale with low cost. For example, reducing the reaction chamber size by a factor of 10 increases the reaction rate by a factor of 100 because the smaller characteristic length of the system decreases diffusion time. In addition to faster reaction times, the amounts of analyte and reagents required are also reduced proportionally to the reduction of the reaction chamber volume. Not only does this reduce the cost of the test by reducing the required amounts of chemicals, it also allows more types of tests to be conducted in parallel with the same size of sample [4].

\subsection{Biochips}

Materials used in microfluidic and biological applications must fulfill a stringent list of requirements. Biocompatibility is probably the most important one. The entire bill of materials need to be able to operate without releasing toxic chemicals, reacting with the fluids being transported and analyzed, or activate unwanted immunological response in the biological systems under analysis [31,32]. Optical transparency is also a requirement for visualization of the flow using a microscope or video camera, enabling the identification and measurement of particles, cells, fluorescence, and the use of other colorimetric detection methods [3].

Biochips are based on the biological recognition and allow forthe simultaneous analysis of a large number of compounds [33]. The discussed technology demands the application of very smallamounts of starting material that minimizes the analysis cost [33]. Due to their advantages, the biochips have been applied for thedetermination of various diagnostic markers [34] and genetic pre-disposition to many diseases [35]. Also, they have been successfullyused in different practical fields such as molecular biology [35], medicine [37], biotechnology [38], etc [39].

The most important part of obtaining the final product is the production and subsequent functionalization of microfluidics by the specified production methods. Because all living things try to adapt to their environment in order to sustain their lives by perceiving the changes in the environment they live in [40]. Advances in basic sciences have enabled the investigation of the functions of other biological materials as well as enzymes [41]. As a result, a wide variety of microfluidic systems have been developed and are being developed.

In microfluidic device studies, enzyme fixation (immobilization) in the biological sensing region of the design is an important step. The most commonly used methods for enzyme immobilization in studies include; arresting in gel, intramolecular crosslinking, adsorption, covalent binding and encapsulation (Fig. 1) [40, 42]. 


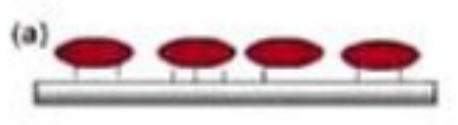

(c)

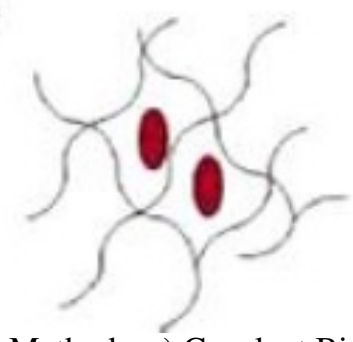

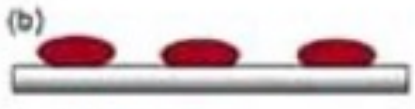

(d)

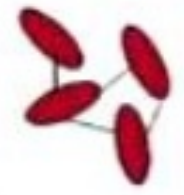

(o)

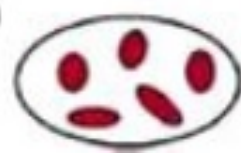

Figure 1. Enzyme Immobilization Methods, a) Covalent Binding, b) Adsorption, c) Arresting in Gel, d) Cross-Linking in Molecules, e) Encapsulation [40].

Baudoin et al. prepared two layer of biochips using polydimethylsiloxane (PDMS, Sylgard 184, Dow Corning, Midland / USA). Briefly, the biochip consists of cell culture chamber manufactured with two layers. The microstructured bottom layer, with series of microchambers and microchannels, is used as a support for cell attachment. The second PDMS layer, with a reservoir (depth of $100 \mu \mathrm{m}$ ), is placed on top of the first layer and includes an inlet and outlet microfluidic network for homogenous culture medium distribution $[43,44]$.

\subsection{Microfabrication}

The fabrication of small scale microfluidic devices relies on high-precision micromachining techniques capable of creating high aspect ratio structures. Micromachining techniques can be basically divided in two major categories: bulk and surface techniques. Bulk techniques rely on the direct modification of a substrate material, usually a monocrystalline silicon wafer, glass, quartz or thick polymer matrix, used as a basic (bulk) material or support to shape the desired structures. Typical substrate thicknesses are in the range of several tens to hundreds of micrometers (Fig. 2 A). On the other hand, surface micromachining techniques involve the deposition of various layers of materials in the form of thin films and their definition into the desired structural shape (Fig. 2 B). Typical thicknesses of the various layers in surface micromachining techniques are in the range of a few nanometers to a few micrometers, much smaller than the bulk thickness, which is used to support the microfluidic device itself [3].
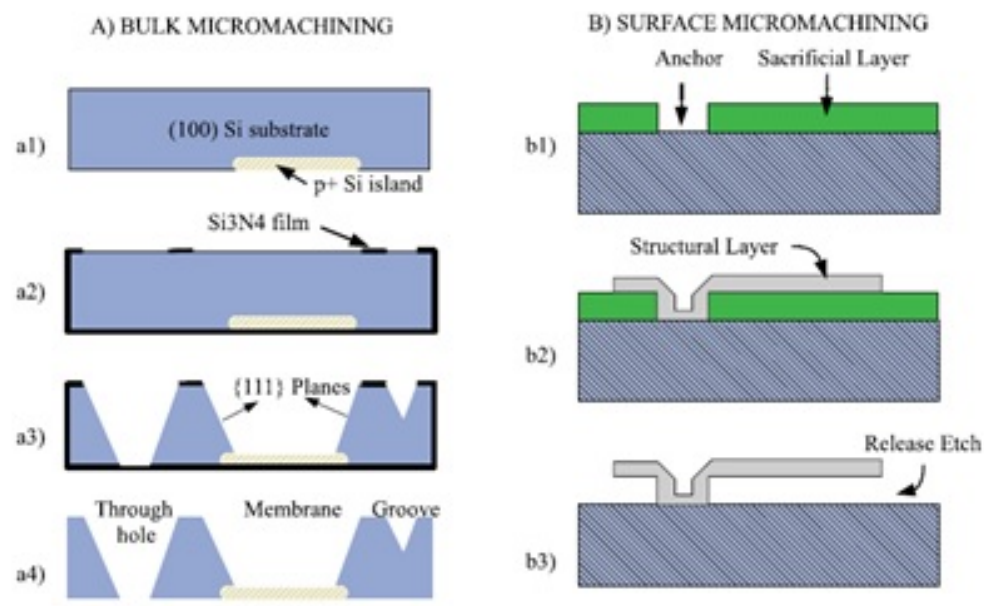

Figure 2. Schematic illustration examples of (a) bulk micromachining process in a silicon substrate and (b) surface micromachining process [3].

\subsection{Microfluidics devices}

These devices are of great interest in physical, chemical, and biological applications. They have stringent specifications in terms of the precision involved in their fabrication as well as in terms of the compatibility between the different materials used to fabricate them and the possible interaction between these parts and the fluids/biological systems of interest. Typical microfluidic systems consist of four main components: (1) microfluidic pumps for driving the fluids along the system (based on various actuation mechanisms), (2) microfluidic valves for controlling and directing the flow as desired, (3) microfluidic channels 
and chambers, which are the passive and the primary fluidic interconnecting components of these systems, and (4) active microfluidic components integrated with closed-loop temperature controllers, optical detectors, electrodes for the application of test voltages and currents, and so forth [3].

\subsection{Fabrication of microfluidics devices}

Most microfluidic devices are fabricated by replica molding techniques [45, 46] because it allows for simple, low-cost prototyping of micro channels. Fig. 3 shows a typical fabrication procedure of a microfluidic chip. The polydimethylsiloxane (PDMS) microchannel is replicated from an SU-8 [47] photoresist negative pattern. Holes for tube connection are then mechanically punched through the channel. The channel is fixed onto a substrate, which often is a glass slide. There are several techniques for bonding the top micro channel and the bottom substrate. Typically, the PDMS part is O2 plasma treated and pressed onto the glass substrate at $100^{\circ} \mathrm{C}$ to create permanent bonding. Simple mechanical clamps are also commonly used. Techniques for microchannel fabrication based on lithography include bulk micromachining of silicon. Anisotropic wet etching $[48,49]$ or DRIE [50, 51] creates grooves on silicon substrates. Other techniques include hot embossing [52] and injection molding [53], which are suitable for mass production [4].

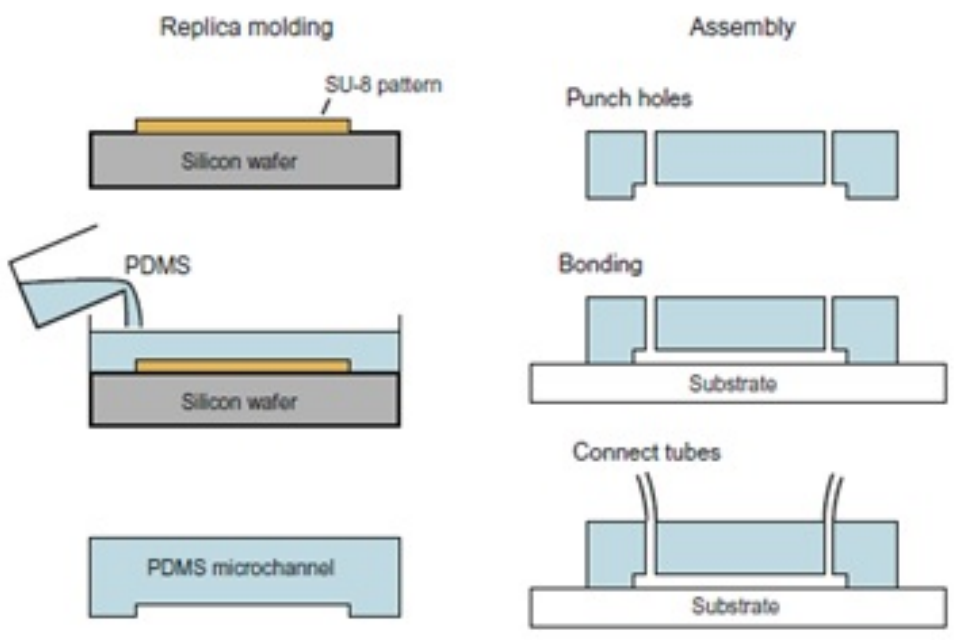

Figure 3. Fabrication of a PDMS microchannel [4].

\subsection{Wearable microfluidics chips}

As a combination of wearable technology and microfluidis, wearable microfluidics directly contacts human skin, by which it enables the collection and analysis of body fluid as well as integration with wireless data transfer function. The wearable microfluidic chips can achieve the real-time continues vital signs monitoring of glucose [54], lactate [55], $\mathrm{Na}^{+} / \mathrm{K}^{+}$[56], $\mathrm{Ca}_{2}^{+}$ [57] and $\mathrm{pH}$ of sweat [58], with the collection and analysis of sweat, interstitial fluid, saliva and tears. For the analysis of glucose and lactate, instead of directly collecting blood, with the help of wearable microfluidics technology, the minimally invasive and non-invasive sample collection methods could be used to ease patients' pain. Besides the advantages in sample collections, other advantages of wearable microfluidics are as follows: (1) the volume of the body fluid sample needed is much less compared with traditional methods; (2) the volume of the reagent needed for each test is also lowered to micro/nano liter scale, which significantly reduces the cost of each analysis; (3) the real-time and continues in vivo monitoring of body fluid and wireless data transfer [59] could be achieved, which is almost impossible for traditional in vitro analysis method; (4) the wearable microfluidics can be easily integrated with other kinds of sensors since the fabrication technology of microfluidics is inherited from the microelectromechanical systems (MEMS). Sensors such as optical sensor, electrochemical sensor, piezoelectric sensor and biosensors can be integrated in the wearable microfluidics chips to meet different requirements of testing body fluid with much less technical barrier [29].

\subsection{Exercise}

Since physical exercise is a factor contributing to the quality of life of people, it is recommended as a very important prescription for both health and disease therapy [60]. The main purpose of exercise; to prevent organic and physical disorders caused by an inactive life and to maintain physical fitness for many years [61]. It is stated that there are physiological, motoric, psychological and sociological benefits in regular sports $[61,62,63]$. According to many studies, the increase in the physical activity of the individuals decreases the risk of death and prolongs the human life [60, 61]. In addition, the type of exercise, intensity, contests, traumas, stress cause both physiological and metabolic changes in the human body [60]. Therefore, the relationship between athletic performance, heavy exercise and physiological changes needs to be well known. 


\section{APPLICATION OF MICROFLUIDICS CHIPS AND BIOCHIPS}

Most of the energy required for the tissue and cell activity is source from the glucose in blood. For the maintenance of organ and tissue functionality, the glucose level should be kept at a certain range. Lactic acid is the final product of glycolysis. In medical filed, it is an important indication for the exercise load control, diabetic monitoring and the detction of sugar souced disease [29]. Currently, the most popular dection methods of blood glucose are either using fully automatic biochemical analyser or fast blood glucose meter [64]. Compared with traditional method for the test of glucose, the wearable microfluidics technology provides a minimally invasive or non-invasive method. The minimally invasive method refers to collection of blood using microneedles array, of which the length of needles would not reach the subcutaneous neural layer, and the subject can hardly feel the pain. Non-invasive method refers to the indirect testing of glucose from the interstitial fluid [65, 66].

Gowers et al. developed a 3D-printed wearable microfluidic system, which could conduct the on-line testing of glucose and lactic acid simultaneously. A minimally invasive method was used with a Food and Drug Administration (FDA)-approved clinical microdialysis probe for the analysis of glucose and lactic acid. Fig. 4 A shows the integration method of the probes and microfluidic chips, Fig. $4 \mathrm{~B}$ and Fig. $4 \mathrm{C}$ illustrate the changes of glucose and lactic acid level during a cycling protocol [67].
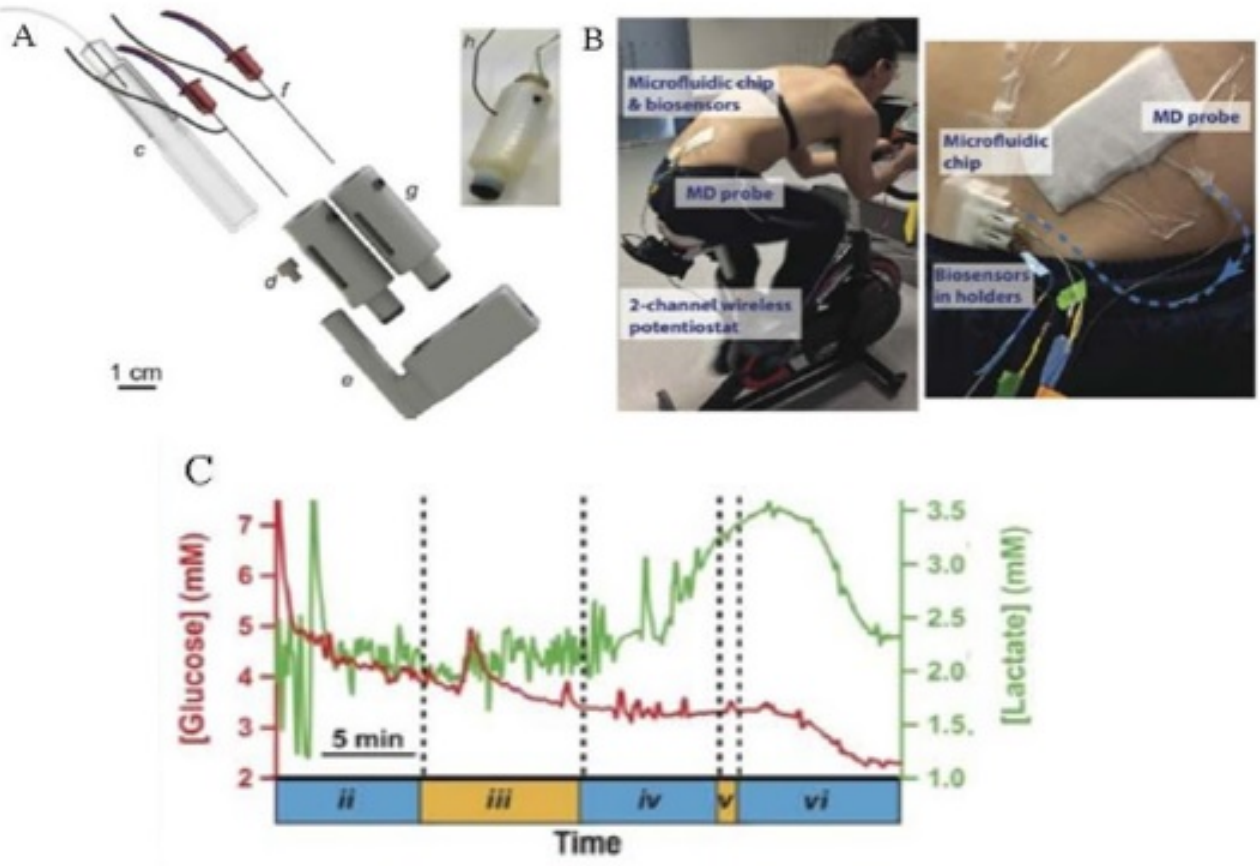

Figure 4. Wearable microfluidics system for glucose and lactic monitoring. (A) microfluidic device for continuous monitoring of dialysate; (B) measure tissue glucose and lactate levels in dialysate during the cycling protocol; (C) glucose and lactate levels during the exercise phase of the cycling protocol [67].

Perspiration is of great importance for maintaining the selfbalancing of inner environment of human body, and is also part of metabolism process. The sweat carries rich information, and real-time continues analysis of sweat is of great significance for the vital life sign monitoring. The $\mathrm{pH}$ value of sweat directly reflects the $\mathrm{pH}$ of human skin [29]. The change of $\mathrm{pH}$ in sweat also reflects the volüme and speed during the perspiration processes, and the $\mathrm{pH}$ of sweat will increase when more sweat is produced [68]. On the basis of measuring $\mathrm{pH}$ value of sweat, we can indirectly speculate the dehydration of the subject, which is useful in the sports science and military field [29].

Traditional methods for testing $\mathrm{pH}$ in sweat require strenuous exercise to produce sweat, and then the sweat sample is collected for the chemical color reaction and the $\mathrm{pH}$ value is read out. This testing method requires the subject to produce significant amount of sweat, and is slow in detection and hard to realize continues real-time monitoring of $\mathrm{pH}$ value of sweat. On the other hand, using wearable microfluidics can realize real-time monitoring of $\mathrm{pH}$ of sweat and consume extra low sample and reagent volüme [29].

Curto et al. proposed a simple and rapid wearable microfluidic devices for the testing of $\mathrm{pH}$ of sweat [58]. They used phosphonium based ionogel integrated with $\mathrm{pH}$ sensitive dyes for the direct testing of $\mathrm{pH}$ in sweat. Compared with other detection methods $[69,70]$, for example, photoelectric sensor to detect chromogenic reaction of $\mathrm{pH}$ in sweat, their method was low-cost with less energy consumption. As shown in Fig. 5 A, the structure of the proposed microfluidic devices was consisted of the absorbent pad for sweat, microchannels for sweat transfer and ionogel sensors for the chromogenic reaction of 
pH in sweat. Fig. 5 B shows the fabrication process of the microfluidic cihps. The multiple layers of polymethyl methacrylate (PMMA), ionogel, and pressure-sensitive adhesive (PSA) were fabricated using $\mathrm{CO}_{2} \mathrm{ffl}$ laser and bonded using PSA. As shown in Fig. $5 \mathrm{C}$, the microfluidics chip was worn on human wrist. During the exercise, the color change of the ionogel could indicate the change of sweat $\mathrm{pH}$ in the range of 4.5 to 8.0 [58].
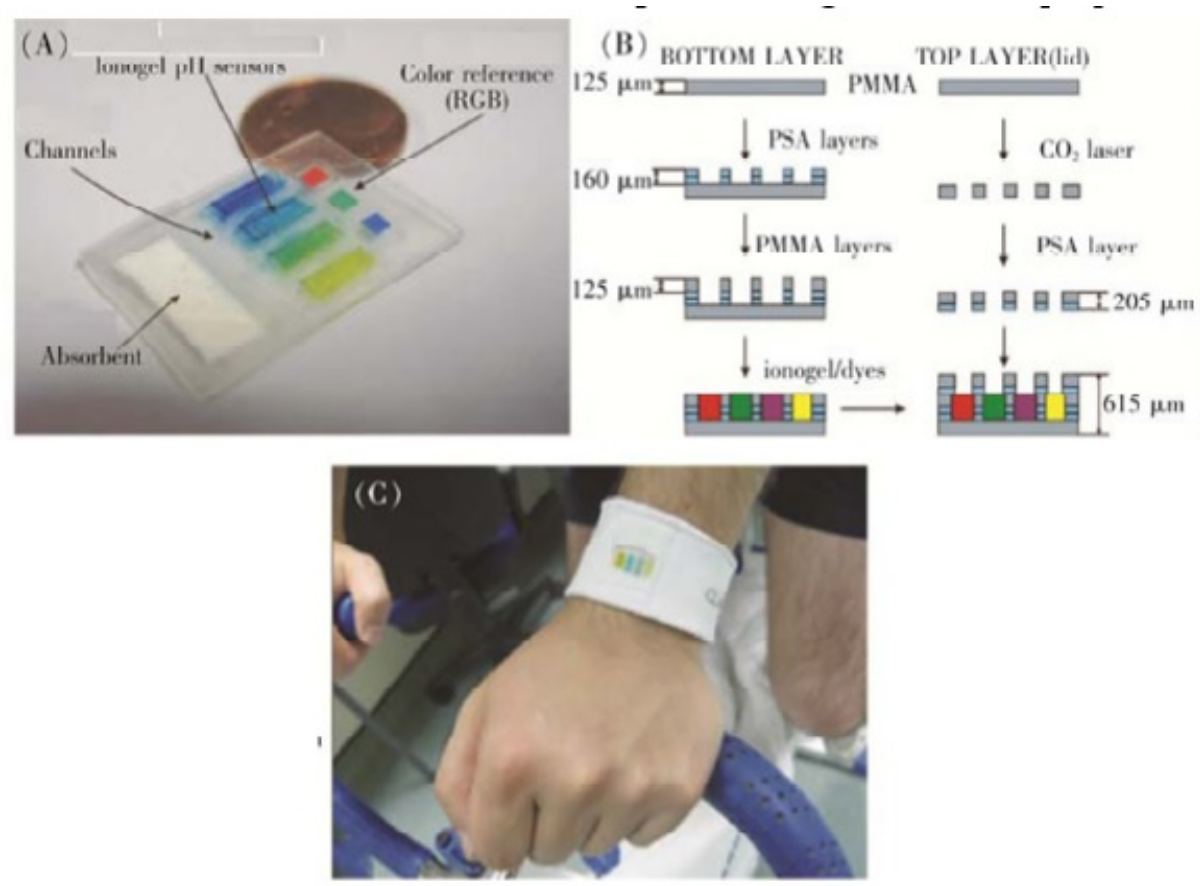

Figure 5. Wearable microfluidic system for sweat pH monitoring. (A) Image of the micro-fluidic platform; (B) micro-fluidic platform fabrication process; (C) image of the micro-fluidic system integrated into a wrist-band [58].

Coyle et al. fabricated a wearable microfluidic system that integrated the textile-based microfluidics for fluid transportation and a series of sensors for the analysis of sweat, including pH sensor [71]. Similar to Coyle's idea, Caldara et al. fabricated a wearable, flexible and non-toxic sweat sensor using the chromogenic reaction of organically modified silicate (ORMOSIL) [72]. As shown in Fig. 6, Tsioris et al. innovatively used the functionalized silk which had chromogenic reaction under different $\mathrm{pH}$ and integrated the silk in a PDMS-based microfluidic device for testing $\mathrm{pH}$ [73].

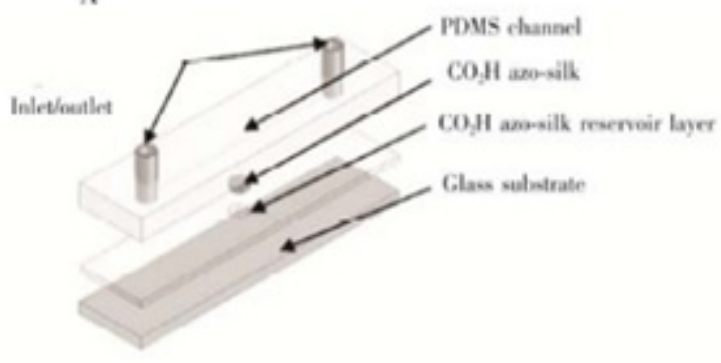

B

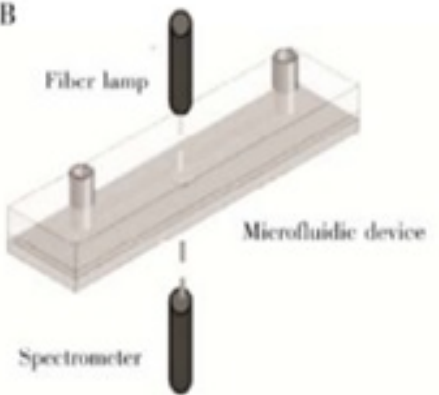

Figure 6. Structure of polydimethylsiloxane (PDMS)-based wearable microfluidic system for sweat $\mathrm{pH}$ monitoring. (A) Schematic of the microfluidic device composed of the PDMS channel, the $\mathrm{CO}_{2} \mathrm{H}$ azo-silk, and an inlet/outlet; (B) optofluidic device setup [73].

$\mathrm{The} \mathrm{Na}^{+}$and $\mathrm{K}^{+}$can directly reflect the hydration of human skin, the dehydration status and losses of electrolytes for the personal (athlete, coal miner, soldier) working in extreme environment (i.e. hot and humid) by monitoring the $\mathrm{N}^{+}$and $\mathrm{K}^{+}$in sweat. In the severe environment with high temperature and humid, the sweating is the major way for the cooling of body, however, the sweating caused loss of electrolytes. If the body cannot adjust the environment well enough, the accumulated loss of $\mathrm{Na}^{+}$(higher than $40 \mathrm{mM}$ ) will finally lead to muscle cramps or even heat stroke. Thus, monitoring the concentration of $\mathrm{Na}^{+}$ and $\mathrm{K}^{+}$is of great importance for the health of people working in severe environment. It is worth to mention that during the 
body fluid testing, the concentrations of $\mathrm{Na}^{+}$and $\mathrm{K}^{+}$are also related to the $\mathrm{pH}$ value of the body fluid. Thus the simultaneous detection of ionic concentration and $\mathrm{pH}$ value is usually required [57].

As shown in Fig. 7, Matzeu et al. assembled dual ion-selective electrodes on the polyethylene terephthalate (PET) layer of a seven-layer microfluidic devices (Fig. 7 A), the polymethyl methacrylate (PMMA) and pressure sensitive adhesive (PSA) layers in this microfluidic chip were fabricated using $\mathrm{CO}_{2}$ laser ablation (Fig. $7 \mathrm{~B}$ ), the PSA layers were used for the bonding between layers [56]. In this study,the researchers analyzed the relations of the output voltage and the concentration of $\mathrm{Na}^{+}$ using different electrode materials in the stationary cycling sessions (Fig. 7 C). A miniaturized wireless system was also developed in this study for the data transfer [56].
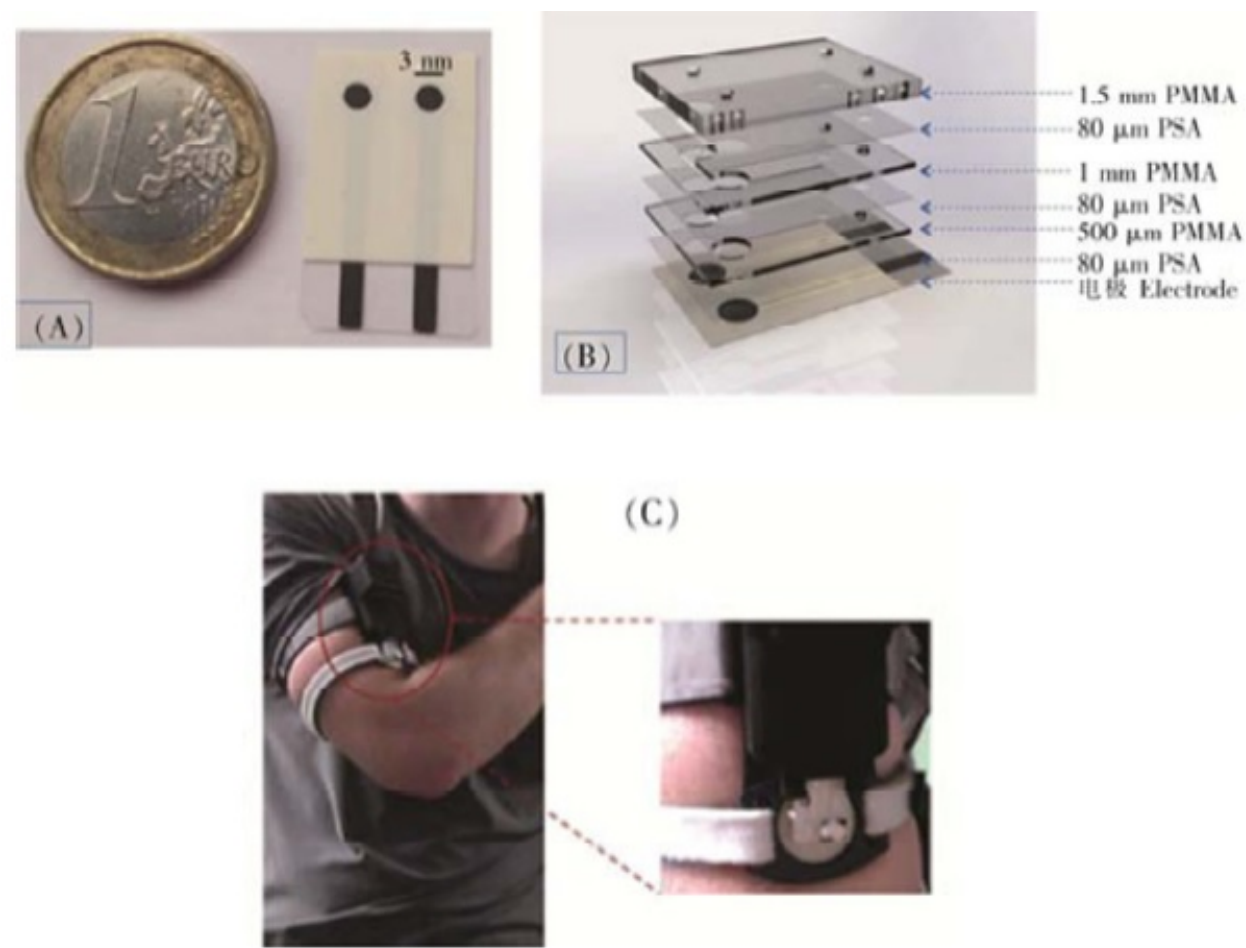

Figure 7. Polymer-based multi-layer wearable microfluidic system for sweat $\mathrm{Na}^{+}$monitoring. (A) Dual screen-printed electrodes on a polyethylene terephthalate (PET) substrate; (B) different layers employed to build up the microfluidics collecting system; (C) microfluidic chip positioned on the upper arm of the subject [56].

Nyein et al. used ion-selective electrodes to detect $\mathrm{Ca}_{2}^{+}$and $\mathrm{pH}$ value in sweat simultaneously [57]. $\mathrm{Ca}_{2}^{+}$detection electrode was made with a combination of organic membrane containing neutral carrier calcium (ETH 129) and an ion-selection transducer. The wearable microfluidic devices were attached to a flexible printed circuit board which handled the single process and wireless data transmission. The sensitivity for $\mathrm{Ca}_{2}^{+}$was as high as $33.7 \mathrm{mV}$ per decade [57].

Ingber et al. developed a microfluidic device which removes magnetized Escherichia coli bacteria from flowing solutions containing red blood cells [74]. The same group also demonstrated a blood cleansing device that removes Candida albicans fungi from flowing human whole blood with over $80 \%$ clearance at a flow rate of $20 \mathrm{~mL} / \mathrm{h}$ [75]. Furdui et al. reported an integrated silicon microchip for separation of Jurkat cells from reconstituted horse blood samples as well as human blood (about 1:10,000 ratio of Jurkat cells to blood cells) [76]. Zborowski et al. demonstrated the separation of MCF7 cells (breast cancer cell line) from mixtures of human leukocytes [77].

\section{CONCLUSIONS}

Wearable microfluidics is a cross-disciplinary field involving fields such as electronics, materials science, biological and analytical chemistry. Advances in these related fields can affect or advance wearable microfluidics. However, currently wearable microfluidics are still at an early stage. Large-scale commercial applications are still waiting to be discovered.

Compared with traditional blood draws for lactate, the epidermal biosensor is noninvasive, is simple-to-operate, and causes no hindrance to the wearer. Future efforts are aimed at further miniaturization and integration of the electronic interface, data processing, and wireless transmission of the results. Moreover, future studies will seek to concurrently correlate lactate levels measured in the perspiration with those measured in the blood during a controlled fitness routine [78]. 
The developed biosensing platform on integration with portable electronics has the potential to be a self-monitoring wearable device for real-time tracking of human lifestyle [79].

Current detection methodsfor $\mathrm{pH}$ value, $\mathrm{Na}+$ and $\mathrm{K}+$ in sweat are focusing on the ion-selective membrane or electrodes, which may experience signal drifting and require frequent calibration. It can be expected that, in the near future, more researchers will turn to the electrochemical method using carbon nanotube (CNT) or graphene. Moreover, with the development of biosensor, integration of wearable microfluidics with biosensor would be expected [29].

From the current researches, we can observe that the wearable microfluidic systems is still at its early stage which is hardly considered the comfort of the wearers. This comfortlessness is mainly due to that the system is still too large and heavy. The whole system may consist of the separated modules for sample collection, analysis, data transfer and power source. The recent tattoo-like wearable microfluidics inspires the desire for the highly integrated wearable microfluidic system: the whole system should be integrated in a single flexible substrate/film, which has excellent biocompatibility, gas permeability and small size. Furthermore, the power source should also seek for some alternatives. A more compact battery or even the energy harvesting system may be integrated in the wearable microfluidic system [29].

\section{REFERENCES}

[1] Feynman, R. P. (1992). There's plenty of room at the bottom [data storage]. Journal of microelectromechanical systems, $1(1), 60-66$.

[2] Moore, G. E. (1998). Cramming more components onto integrated circuits. Proceedings of the IEEE, 86(1), 82-85.

[3] Zahn, J. D. (2010). Methods in Bioengineering Biomicrofabrication and Biomicrofluidics, Artech House, ISBN-13: 978-1-59693-400-9.

[4] Zhang, J. X., \& Hoshino, K. (2018). Molecular Sensors and Nanodevices: Principles, Designs and Applications in Biomedical Engineering. Academic Press.

[5] Wang, J., Pumera, M., Chatrathi, M. P., Escarpa, A., Konrad, R., Griebel, A., Dörner, W., \& Löwe, H. (2002). Towards disposable lab-on-a chip: Poly (methylmethacrylate) microchip electrophoresis device with electrochemical detection. Electrophoresis, 23(4), 596-601.

[6] Chin, C. D., Linder, V., \& Sia, S. K. (2007). Lab-on-a-chip devices for global health: Past studies and future opportunities. Lab on a Chip, 7(1), 4157.

[7] Madou, M. J. (2011). Manufacturing techniques for microfabrication and nanotechnology (Vol. 2). CRC press.

[8] Rebeiz, G. M. (2004). RF MEMS: theory, design, and technology. John Wiley \& Sons.

[9] Neukermans, A., \& Ramaswami, R. (2001). MEMS technology for optical networking applications. IEEE Communications Magazine, 39(1), 62-69.

[10] Lin, L. Y., \& Goldstein, E. L. (2002). Opportunities and challenges for MEMS in lightwave communications. IEEE Journal of selected topics in quantum electronics, 8(1), 163-172.

[11] Wang, W., \& Soper, S. A. (2006). Bio-MEMS: technologies and applications. CRC press.

[12] Tabeling, P. (2005). Introduction to microfluidics. Oxford University Press on Demand.

[13] Mastrangelo, C. H., Burns, M. A., \& Burke, D. T. (1998). Microfabricated devices for genetic diagnostics. Proceedings of the IEEE, 86(8), 1769-1787.

[14] Santini, Jr, J. T., Richards, A. C., Scheidt, R., Cima, M. J., \& Langer, R. (2000). Microchips as controlled drugdelivery devices. Angewandte Chemie International Edition, 39(14), 2396-2407.

[15] Thomas, D. J., Tehrani, Z., \& Redfearn, B. (2016). 3-D printed composite microfluidic pump for wearable biomedical applications. Additive Manufacturing, 9, 30-38. 
[16] Su, W., Cook, B. S., \& Tentzeris, M. M. (2016). Additively manufactured microfluidics-based "peel-and-replace" RF sensors for wearable applications. IEEE Transactions on Microwave Theory and Techniques, 64(6), 1928-1936.

[17] Raya, R., Roa, J. O., Rocon, E., Ceres, R., \& Pons, J. L. (2010). Wearable inertial mouse for children with physical and cognitive impairments. Sensors and Actuators A: Physical, 162(2), 248-259.

[18] Lin, C. S., Hsu, H. C., Chiu, C. C., Lin, S. L., \& Chao, C. S. (2006). A PDA based wearable system for real-time monitoring of human falls. IETE journal of research, 52(6), 403-416.

[19] Georga, E. I., Protopappas, V. C., Bellos, C. V., \& Fotiadis, D. I. (2014). Wearable systems and mobile applications for diabetes disease management. Health and Technology, 4(2), 101-112.

[20] Hsu, Y. L., Chung, P. C., Wang, W. H., Pai, M. C., Wang, C. Y., Lin, C. W., Wu, H. L., \& Wang, J. S. (2014). Gait and balance analysis for patients with Alzheimer's disease using an inertial-sensor-based wearable instrument. IEEE journal of biomedical and health informatics, 18(6), 1822-1830.

[21] Mukhopadhyay, S. C. (2014). Wearable sensors for human activity monitoring: A review. IEEE sensors journal, 15(3), 1321-1330.

[22] Attal, F., Mohammed, S., Dedabrishvili, M., Chamroukhi, F., Oukhellou, L., \& Amirat, Y. (2015). Physical human activity recognition using wearable sensors. Sensors, 15(12), 31314-31338.

[23] Taylor, G. S., \& Barnett, J. S. (2013). Evaluation of wearable simulation interface for military training. Human factors, 55(3), 672-690.

[24] Venkatraman, S., \& Yuen, S. G. J. (2015). U.S. Patent No. 8,998,815. Washington, DC: U.S. Patent and Trademark Office.

[25] Yang, J., Wei, D., Tang, L., Song, X., Luo, W., Chu, J., Gao, T., Shi, H., \& Du, C. (2015). Wearable temperature sensor based on graphene nanowalls. Rsc Advances, 5(32), 25609-25615.

[26] He, H., Li, Y., Guan, Y., \& Tan, J. (2015). Wearable ego-motion tracking for blind navigation in indoor environments. IEEE Transactions on Automation Science and Engineering, 12(4), 1181-1190.

[27] Villa, F., Magnani, A., Maggioni, M., Stahn, A., Rampichini, S., Merati, G., \& Castiglioni, P. (2016). Wearable multi-frequency and multi-segment bioelectrical impedance spectroscopy for unobtrusively tracking body fluid shifts during physical activity in real-field applications: a preliminary study. Sensors, 16(5), 673.

[28] Karuei, I., Schneider, O. S., Stern, B., Chuang, M., \& MacLean, K. E. (2014). RRACE: Robust realtime algorithm for cadence estimation. Pervasive and Mobile Computing, 13, 52-66.

[29] Yi-Qiang, F. A. N., Feng, G. A. O., Mei, W., Zhuang, J., Gang, T., \& Zhang, Y. J. (2017). Recent development of wearable microfluidics applied in body fluid testing and drug delivery. Chinese Journal of Analytical Chemistry, 45(3), 455-463.

[30] Taylor, R. F., \& Schultz, J. S. (Eds.). (1996). Handbook of chemical and biological sensors. CRC Press.

[31] Williams, D. (2003). Revisiting the definition of biocompatibility. Medical device technology, 14(8), 10-13.

[32] Freitas Jr, R. A. (2003). Nanomedicine, Vol. IIA: Biocompatibility. Karger Publishers.

[33] Sutandy, F. R., Qian, J., Chen, C. S., \& Zhu, H. (2013). Overview of protein microarrays. Current protocols in protein science, 72(1), 27-1.

[34] Feyzkhanova, G. U., Filippova, M. A., Talibov, V. O., Dementieva, E. I., Maslennikov, V. V., Reznikov, Y. P., Of- 
fermann, N., Zasedatelev, A. S., Rubina, A. Y., \& Fooke-Achterrath, M. (2014). Development of hydrogel biochip for in vitro allergy diagnostics. Journal of immunological methods, 406, 51-57.

[35] Chang, Y. T., Yeh, Y. S., Ma, C. J., Huang, C. W., Tsai, H. L., Huang, M. Y., Cheng, T. L., \& Wang, J. Y. (2017). Optimization of a multigene biochip for detection of relapsed and early relapsed colorectal cancer. journal of surgical research, 220, 427-437.

[36] Leclerc, E., Hamon, J., Legendre, A., \& Bois, F. Y. (2014). Integration of pharmacokinetic and NRF2 system biology models to describe reactive oxygen species production and subsequent glutathione depletion in liver microfluidic biochips after flutamide exposure. Toxicology in vitro, 28(7), 1230-1241.

[37] Kemmler, M., Sauer, U., Schleicher, E., Preininger, C., \& Brandenburg, A. (2014). Biochip point-of-care device for sepsis diagnostics. Sensors and Actuators B: Chemical, 192, 205-215.

[38] Chiang, Y. C., Wang, H. H., Ramireddy, L., Chen, H. Y., Shih, C. M., Lin, C. K., \& Tsen, H. Y. (2018). Designing a biochip following multiplex polymerase chain reaction for the detection of Salmonella serovars Typhimurium, Enteritidis, Infantis, Hadar, and Virchow in poultry products. journal of food and drug analysis, 26(1), 58-66.

[39] Volokitina, M., Krutyakova, M., Sirotov, V., Larionov, M., Tennikova, T., \& Korzhikova-Vlakh, E. (2019). Protein biochips based on macroporous polymer supports: Material properties and analytical potential. Journal of pharmaceutical and biomedical analysis, 165, 242-250.

[40] Aydın, Y. (2012). İletken polimerlerin içerisinde enzim tutuklamasıyla yapılan biyosensörler (Yüksek Lisans Tezi, Karamanoğlu Mehmetbey Üniversitesi, Fen Bilimleri Enstitüsü).

[41] Sassolas, A., Blum, L. J., \& Leca-Bouvier, B. D. (2012). Immobilization strategies to develop enzymatic biosensors. Biotechnology advances, 30(3), 489-511.

[42] Gulrajani, M. L., \& Deepti, G. (2011). Emerging techniques for functional finishing of textiles.

[43] Baudoin, R., Griscom, L., Prot, J. M., Legallais, C., \& Leclerc, E. (2011). Behavior of HepG2/C3A cell cultures in a microfluidic bioreactor. Biochemical Engineering Journal, 53(2), 172-181.

[44] Jellali, R., Paullier, P., Fleury, M. J., \& Leclerc, E. (2016). Liver and kidney cells cultures in a new perfluoropolyether biochip. Sensors and Actuators B: Chemical, 229, 396-407.

[45] Whitesides, G. M. (2006). The origins and the future of microfluidics. Nature, 442(7101), 368.

[46] McDonald, J. C., Duffy, D. C., Anderson, J. R., Chiu, D. T., Wu, H., Schueller, O. J., \& Whitesides, G. M. (2000). Fabrication of microfluidic systems in poly (dimethylsiloxane). ELECTROPHORESIS: An International Journal, 21(1), 27-40.

[47] Lorenz, H., Despont, M., Fahrni, N., LaBianca, N., Renaud, P., \& Vettiger, P. (1997). SU-8: a low-cost negative resist for MEMS. Journal of Micromechanics and Microengineering, 7(3), 121.

[48] Haneveld, J., Jansen, H., Berenschot, E., Tas, N., \& Elwenspoek, M. (2003). Wet anisotropic etching for fluidic 1D nanochannels. Journal of micromechanics and microengineering, 13(4), S62.

[49] Nilsson, A., Petersson, F., Jönsson, H., \& Laurell, T. (2004). Acoustic control of suspended particles in micro fluidic chips. Lab on a Chip, 4(2), 131-135.

[50] Jiang, L., Mikkelsen, J., Koo, J. M., Huber, D., Yao, S., Zhang, L., Zhou, P., Maveety, J. G., Prasher, R., Santiago, J. G., Goodson, K. E., \& Kenny, T. W. (2002). Closed-loop electroosmotic microchannel cooling system for VLSI circuits. IEEE Transactions on Components and Packaging Technologies, 25(3), 347-355.

[51] Di Carlo, D., Jeong, K. H., \& Lee, L. P. (2003). Reagentless mechanical cell lysis by nanoscale barbs in microchannels for 
sample preparation. Lab on a Chip, 3(4), 287-291.

[52] Becker, H., \& Heim, U. (2000). Hot embossing as a method for the fabrication of polymer high aspect ratio structures. Sensors and Actuators A: Physical, 83(1-3), 130-135.

[53] McCormick, R. M., Nelson, R. J., Alonso-Amigo, M. G., Benvegnu, D. J., \& Hooper, H. H. (1997). Microchannel electrophoretic separations of DNA in injection-molded plastic substrates. Analytical Chemistry, 69(14), 2626-2630.

[54] Pu, Z., Zou, C., Wang, R., Lai, X., Yu, H., Xu, K., \& Li, D. (2016). A continuous glucose monitoring device by graphene modified electrochemical sensor in microfluidic system. Biomicrofluidics, 10(1), 011910.

[55] Kim, J., Valdés-Ramírez, G., Bandodkar, A. J., Jia, W., Martinez, A. G., Ramírez, J., Mercier, P., \& Wang, J. (2014). Non-invasive mouthguard biosensor for continuous salivary monitoring of metabolites. Analyst, 139(7), 1632-1636.

[56] Matzeu, G., O’Quigley, C., McNamara, E., Zuliani, C., Fay, C., Glennon, T., \& Diamond, D. (2016). An integrated sensing and wireless communications platform for sensing sodium in sweat. Analytical Methods, 8(1), 64-71.

[57] Nyein, H. Y. Y., Gao, W., Shahpar, Z., Emaminejad, S., Challa, S., Chen, K., Fahad, H. M., Tai, L. C., Ota, H., Davis, R. W., \& Javey, A. (2016). A wearable electrochemical platform for noninvasive simultaneous monitoring of $\mathrm{Ca} 2+$ and pH. ACS nano, 10(7), 7216-7224.

[58] Curto, V. F., Fay, C., Coyle, S., Byrne, R., O’Toole, C., Barry, C., Hughes, S., Moyna, N., Diamond, D., \& Benito-Lopez, F. (2012). Real-time sweat $\mathrm{pH}$ monitoring based on a wearable chemical barcode micro-fluidic platform incorporating ionic liquids. Sensors and Actuators B: Chemical, 171, 1327-1334.

[59] Liu, G., Ho, C., Slappey, N., Zhou, Z., Snelgrove, S. E., Brown, M., Grabinski, A., Guo, X., Chen, Y., Miller, K., Kaya, T.,\& Edwards, J. (2016). A wearable conductivity sensor for wireless real-time sweat monitoring. Sensors and Actuators B: Chemical, 227, 35-42.

[60] Laranjeiro, R., Harinath, G., Burke, D., Braeckman, B. P., \& Driscoll, M. (2017). Single swim sessions in C. elegans induce key features of mammalian exercise. BMC biology, 15(1), 30.

[61] Hartman, J. H., Smith, L. L., Gordon, K. L., Laranjeiro, R., Driscoll, M., Sherwood, D. R., \& Meyer, J. N. (2018). Swimming exercise and transient food deprivation in Caenorhabditis elegans promote mitochondrial maintenance and protect against chemical-induced mitotoxicity. Scientific reports, 8(1), 8359.

[62] Asthana, J., Yadav, A. K., Pant, A., Pandey, S., Gupta, M. M., \& Pandey, R. (2015). Specioside ameliorates oxidative stress and promotes longevity in Caenorhabditis elegans. Comparative Biochemistry and Physiology Part C: Toxicology \& Pharmacology, 169, 25-34.

[63] Chuang, H. S., Kuo, W. J., Lee, C. L., Chu, I. H., \& Chen, C. S. (2016). Exercise in an electrotactic flow chamber ameliorates age-related degeneration in Caenorhabditis elegans. Scientific reports, 6, 28064.

[64] Bruen, D., Delaney, C., Florea, L., \& Diamond, D. (2017). Glucose sensing for diabetes monitoring: recent developments. Sensors, 17(8), 1866.

[65] Rebrin, K., Sheppard, N. F., \& Steil, G. M. (2010). Interstitial Fluid Physiology as it Relates to Glucose Monitoring Technologies: Use of Subcutaneous Interstitial Fluid Glucose to Estimate Blood Glucose: Revisiting Delay and Sensor Offset. Journal of Diabetes Science and Technology, 4(5), 1087-1098.

[66] Keenan, D. B., Mastrototaro, J. J., Weinzimer, S. A., \& Steil, G. M. (2013). Interstitial fluid glucose time-lag correction for real-time continuous glucose monitoring. Biomedical signal processing and control, 8(1), 81-89.

[67] Gowers, S. A., Curto, V. F., Seneci, C. A., Wang, C., Anastasova, S., Vadgama, P., Yang, G.Z., \& Boutelle, M. G. (2015). 3D printed microfluidic device with integrated biosensors for online analysis of subcutaneous human microdialysate. 
Analytical chemistry, 87(15), 7763-7770.

[68] Granger, D., Marsolais, M., Burry, J., \& Laprade, R. (2003). Na+/H+ exchangers in the human eccrine sweat duct. American Journal of Physiology-Cell Physiology, 285(5), C1047-C1058.

[69] Schyrr, B., Pasche, S., Scolan, E., Ischer, R., Ferrario, D., Porchet, J. A., \& Voirin, G. (2014). Development of a polymer optical fiber $\mathrm{pH}$ sensor for on-body monitoring application. Sensors and Actuators B: Chemical, 194, 238-248.

[70] Yan, L., Chang, Y. N., Yin, W., Liu, X., Xiao, D., Xing, G., Zhao, L., Gu, Z., \& Zhao, Y. (2014). Biocompatible and flexible graphene oxide/upconversion nanoparticle hybrid film for optical $\mathrm{pH}$ sensing. Physical Chemistry Chemical Physics, 16(4), 1576-1582.

[71] Coyle, S., Lau, K. T., Moyna, N., O’Gorman, D., Diamond, D., Di Francesco, F., Costanzo, D., Salvo, P., Trivella, M. G., De Rossi, D. E., \& Taccini, N. (2010). BIOTEX-Biosensing textiles for personalised healthcare management. IEEE Transactions on Information Technology in Biomedicine, 14(2), 364-370.

[72] Caldara, M., Colleoni, C., Guido, E., Re, V., \& Rosace, G. (2016). Optical monitoring of sweat pH by a textile fabric wearable sensor based on covalently bonded litmus-3-glycidoxypropyltrimethoxysilane coating. Sensors and Actuators B: Chemical, 222, 213-220.

[73] Tsioris, K., Tilburey, G. E., Murphy, A. R., Domachuk, P., Kaplan, D. L., \& Omenetto, F. G. (2010). Functionalized-SilkBased Active Optofluidic Devices. Advanced Functional Materials, 20(7), 1083-1089.

[74] Xia, N., Hunt, T. P., Mayers, B. T., Alsberg, E., Whitesides, G. M., Westervelt, R. M., \& Ingber, D. E. (2006). Combined microfluidic-micromagnetic separation of living cells in continuous flow. Biomedical microdevices, 8(4), 299.

[75] Yung, C. W., Fiering, J., Mueller, A. J., \& Ingber, D. E. (2009). Micromagnetic-microfluidic blood cleansing device. Lab on a Chip, 9(9), 1171-1177.

[76] Furdui, V. I., Kariuki, J. K., \& Harrison, D. J. (2003). Microfabricated electrolysis pump system for isolating rare cells in blood. Journal of Micromechanics and Microengineering, 13(4), S164.

[77] Fang, B., Zborowski, M., \& Moore, L. R. (1999). Detection of rare MCF-7 breast carcinoma cells from mixtures of human peripheral leukocytes by magnetic deposition analysis. Cytometry: The Journal of the International Society for Analytical Cytology, 36(4), 294-302.

[78] Jia, W., Bandodkar, A. J., Valdes-Ramirez, G., Windmiller, J. R., Yang, Z., Ramirez, J., Chan, G., \& Wang, J. (2013). Electrochemical tattoo biosensors for real-time noninvasive lactate monitoring in human perspiration. Analytical chemistry, 85(14), 6553-6560.

[79] Bhide, A., Cheeran, S., Muthukumar, S., \& Prasad, S. (2019). Enzymatic Low Volume Passive Sweat Based Assays for Multi-Biomarker Detection. Biosensors, 9(1), 13. 